\title{
Sub-arcsecond Mid-infrared Images of NGC 7027 and $\mathrm{BD}+30^{\circ} 3639$
}

\author{
Kevin Volk, Sun Kwok \\ Department of Physics and Astronomy, University of Calgary, Calgary, \\ Alberta, Canada T2N $1 N_{4}$
}

\section{Introduction}

Many carbon-rich PNe show strong emission features due to various stretching and bending modes of aromatic hydrocarbon bonds (AHB). Comparisons between ISO spectra of evolved carbon stars, proto-planetary nebulae (PPNe), and PNe have shown that the AHB features develop late in the PPN phase (Kwok et al. 1999). In order to test the formation history of this carbonaceous component, it would be desirable to image the spatial distributions of the dust components. The Gemini Telescopes, optimized for infrared observations with adaptive optics capabilities, offer the unique opportunity for such observations. In this paper, we present the preliminary results of imaging of NGC 7027 and $\mathrm{BD}+30^{\circ} 3639$ with the OSCIR mid-infrared camera at Gemini North.

\section{Observations and results}

Observations were made with the broad-band $\mathrm{N}$ filter and with four narrower band filters $(10.3,11.7,12.5$, and $20.8 \mu \mathrm{m})$. Observations to form five-point maps in the $11.7 \mu \mathrm{m}$ filter were taken for both objects. For NGC 7027, a similar five-point map in the $\mathrm{N}$-band filter was carried out (Fig. 1). The image resolution was $0.4^{\prime \prime}$ in the $10 \mu \mathrm{m}$ window. The images were absolutely calibrated using the ISO spectra of these two nebulae.

Since both nebulae show strong AHB emissions, it is possible to locate the emission regions of the $11.3 \mu \mathrm{m}$ AHB feature by subtracting the (scaled) adjacent continuum image from the $11.7 \mu \mathrm{m}$ image. A comparison between the ISO spectrum and the OSCIR profiles shows that the $11.3 \mu \mathrm{m}$ feature fits in almost entirely within the $11.7 \mu \mathrm{m}$ filter and the continuum can be well approximated by the $10.3 \mu \mathrm{m}$ filter for BD $+30^{\circ} 3639$. For NGC 7027 the $12.5 \mu \mathrm{m}$ filter is used for the continuum, since the $12.8 \mu \mathrm{m}$ [NeII] line is weak while the $10.5 \mu \mathrm{m}$ [Srv] line is strong. In both objects the feature appears to be emitted in the region just outside of the ionized region. This suggests that the change in the spectral character of the aromatic emission features through the PPN phase and into the PN phase may well reflect a destruction of the carrier molecules in the ionized region along with the changing excitation conditions.

\section{References}

Kwok, S., Volk, K., and Hrivnak, B.J. 1999, A\&A, 350, L35 

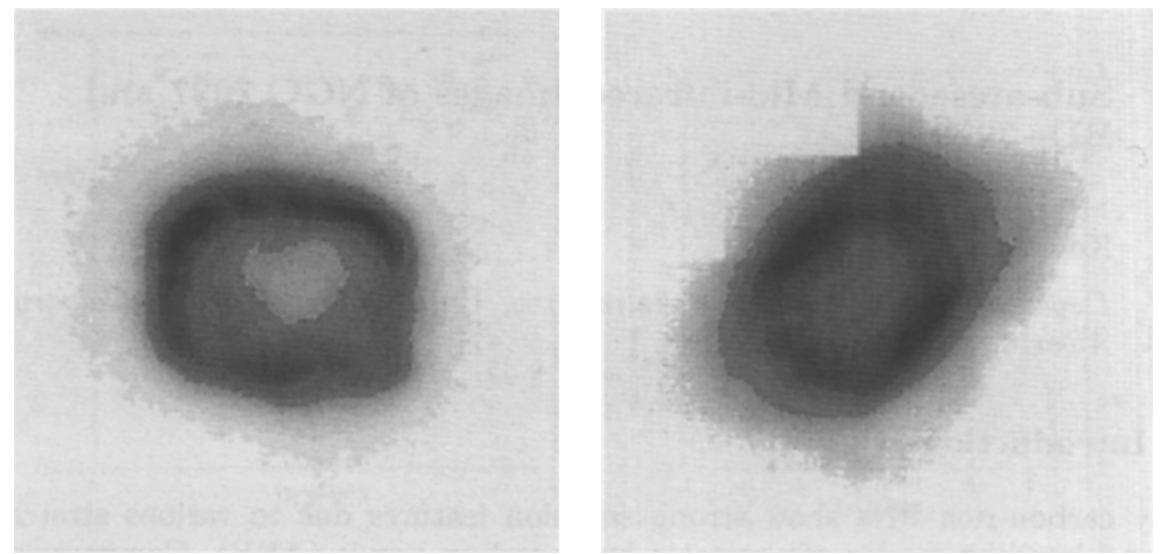

Figure 1. Left: the $11.7 \mu \mathrm{m}$ 5-point mosaic image of $\mathrm{BD}+30^{\circ} 3639$. Right: the N-band 5-point mosaic image of NGC 7027.

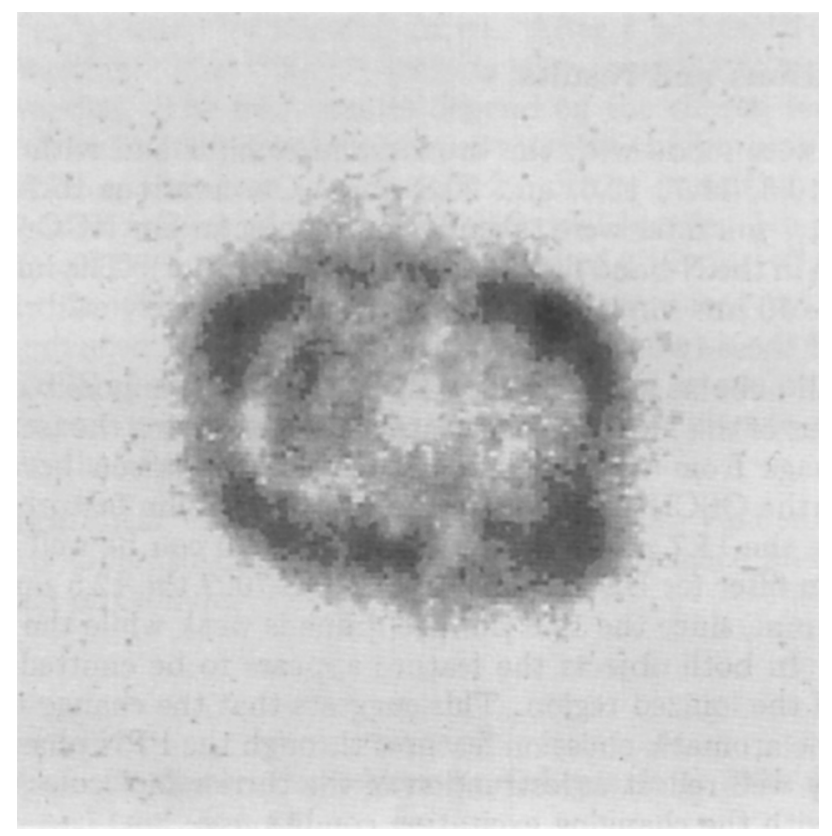

Figure 2. The absolute flux calibrated $11.7 \mu \mathrm{m}$ image of $\mathrm{BD}+30^{\circ} 3639$ with continuum subtracted, showing that the AHB emission arises mostly outside of the ionized region. 